\title{
Electrical shielding for silicon nanowire biosensor in microchannels
}

\author{
Songyue Chen, ${ }^{1, a)}$ Yanbo Xie, ${ }^{1}$ Arpita De,${ }^{1}$ Albert van den Berg, ${ }^{1}$ and Edwin T. Carlen ${ }^{1,2}$ \\ ${ }^{1}$ MESA+ Institute for Nanotechnology, University of Twente, $7522 \mathrm{NH}$ Enschede, The Netherlands \\ ${ }^{2}$ Institute of Materials Science, University of Tsukuba, Tsukuba 305-8573, Japan
}

(Received 6 September 2013; accepted 9 October 2013; published online 23 October 2013)

\begin{abstract}
When integrating silicon nanowire biosensors with a microfluidic sample delivery system, additional challenges are introduced. Noise and erroneous signal generation induced by sample fluidic handling such as flow rate fluctuations during sample switching reduce the quality and reliability of the measurement system. In this paper, we propose an effective electrical shielding method to improve the stability and reliability of the setup by placing double electrodes instead of a single electrode that is traditionally used for nanowire sensors. Experimental results show that with proper shielding electrical measurements are not influenced by flow speed variations or during sample switching. @ 2013 AIP Publishing LLC. [http://dx.doi.org/10.1063/1.4826689]
\end{abstract}

Silicon nanowire (Si-NW) field-effect transistors (FETs) can perform label-free real-time detection of biomolecules at low detection limits. ${ }^{1-3}$ The multigate structure renders the sensors extremely sensitive to small surface potential changes over the gate oxide, due to association or dissociation of charged ions or charged molecules. ${ }^{4}$ There are quite a few practical challenges associated with integrated nanoscale biosensors, such as the intrinsic charge detection in electrolyte solution, ${ }^{5,6}$ stable sample transport, ${ }^{7}$ and effective solution gating. ${ }^{8,9}$ Biomolecule sensing with Si-NW FET sensors requires a low ionic strength solution in order to be able to detect intrinsic charge of biomolecules within Debye length $\lambda_{\mathrm{D}}$, e.g., $1 \mathrm{mM}$ salt solution for $\lambda_{\mathrm{D}} \approx 9.7 \mathrm{~nm}$ and $100 \mathrm{mM}$ salt solution for $\lambda_{\mathrm{D}} \approx 1 \mathrm{~nm}$. On the other hand, in many cases, a high sample flow speed $(>0.5 \mathrm{~mm} / \mathrm{s})$ is required so that the sample transport is not limited by diffusion but rather by the reaction time of target-probe complex on the sensor surface. ${ }^{10}$ Diffusion limited sample transport to the NW surface, especially at very low sample concentration, can take hours or even days to reach equilibrium. ${ }^{11}$ However, both low ionic strength solution and high flow rate contribute to a significant level of streaming current near the surface of the microchannel that is generated by the transport of counterions in the electric double layer at the interface of the microchannel and the electrolyte solution. ${ }^{12}$ This redistribution of counterions also affects the local surface potential of the Si-NW gate oxide and thus generates undesirable conductance change in the Si-NW. The utilization of Si-NW as flow speed sensor has been demonstrated by measuring the conductance change of Si-NW FETs. ${ }^{13}$ Thus, the switching of solution and/or variation of flow rate can both influence the charge distribution over the sensor surface, thus produce significant current noise from Si-NW sensor system. ${ }^{14}$ Therefore, for biomolecule detection, the effect of the streaming potential change over the gate oxide should be minimized in order to improve the detection limit and perform stable and reliable measurements. We propose an effective and simple method to shield the sample solution for nanosensing of charged

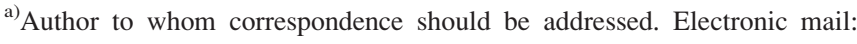
s.chen@utwente.nl.
}

biomolecules inside microchannel: use of double Pt electrodes, while one Pt electrode is placed in upstream and the other one is placed in downstream. Pt is used as an electrode because it can be easily integrated with Si-NW chips. We show that the surface potential fluctuation caused by flow rate change and valve switching can be greatly reduced.

$\mathrm{Si}-\mathrm{NW}$ devices are fabricated with the previously reported method ${ }^{15}$ that uses conventional microlithography, wet anisotropic etching, oxidation, and dry etching. All SiNW sensors are boron doped with $N_{\mathrm{a}} \sim 10^{18} \mathrm{~cm}^{-3}$ with height $100 \mathrm{~nm}$ and length $100 \mu \mathrm{m}$. Each NW device consists of an array of $100 \mathrm{Si}-\mathrm{NWs}$. Si-NW chips have dimension of $2 \mathrm{~cm}^{2}$ and are insulated with polyimide layer with patterned windows that expose the sensors to the sample solution. Prior to each measurement, Si-NW chips are treated with UV/ozone (UV/Ozone ProCleaner Plus, Bioforce Nanoscience) for 5 min to ensure a clean gate oxide surface. The polydimethylsiloxane (PDMS) microfluidic chips are fabricated with a silicon mold, with $50 \mu \mathrm{m}$ height, $200 \mu \mathrm{m}$ width, and length of $1.2 \mathrm{~cm}$, and are cleaned with ethanol before bonding to the Si-NW chips. All experiments are performed with a $1 \mathrm{mM}$ phosphate buffered solution in $1 \mathrm{mM} \mathrm{NaCl}$ solution and $\mathrm{pH}$ 7.3 with $\lambda_{\mathrm{D}} \approx 7 \mathrm{~nm}$. The sample solution is delivered by pressure source (MFCS-8C, Fluigent) and the sample switch is realized with an injection valve (Cheminert Nanovolume 10-port valve, Valco Instruments Co. Inc.). More details of sample delivery and NW sensing system can be found in Ref. 14. The flow rate is controlled by the pressure source, the inner diameter and the length of Polyetheretherketone (PEEK) tubings, and size of the microchannel. A 100 millibars applied pressure corresponds to a flow speed of $V_{\text {flow }} \approx 2.4 \mathrm{~mm} / \mathrm{s}$. Figure 1 shows the diagram of Si-NW sensor inside microfluidic chip. The Si-NW array is located $3 \mathrm{~mm}$ from outlet and $9 \mathrm{~mm}$ from inlet for the present measurements. The Pt tubes are used to connect PEEK tubing to the microchannel for both inlet and outlet and in the meantime to provide solution potential control over the microchannel. The two Pt tubes can be replaced by two patterned Pt electrodes on chip adjacent to NW sensor. The electrical measurements of Si-NW sensor are conducted with a lock-in-amplifier (SR 830, Stanford Research Systems) at drain-source voltage $v_{\mathrm{ds}}=10 \mathrm{mV}$ and frequency $f=30 \mathrm{~Hz}$ and $V_{\mathrm{bg}}=0 \mathrm{~V}$. 


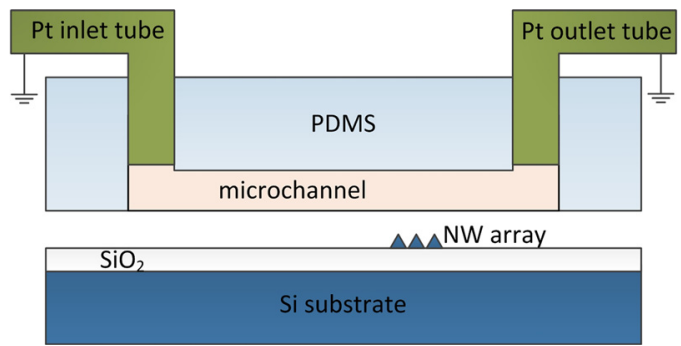

FIG. 1. Schematic of integrated Si-NW microfluidic chip.

When there is pressure-driven flow inside the microchannel, a longitudinal potential gradient is established between the Si-NW gate oxide and the grounding point inside the microchannel. An equivalent circuit diagram of the integrated Si-NW microfluidic chip is depicted in Fig. 2. The streaming current $I_{\text {str }}$ is a function of flow rate, electrolyte ionic strength, charge density of microfluidic channel, and the cross-sectional dimension of the channel. With a fixed microchannel size, the $I_{\text {str }}$ can be decreased by reducing the flow rate, using higher ionic strength solution, and/or decrease the surface charge distribution, e.g., using solution with lower $\mathrm{pH}$ values. ${ }^{12}$ During the experiments, $I_{\text {str }}$ is regarded to be constant with fixed flow rate and is opposite to the direction of streaming flow due to the negatively charged walls of PDMS microchannel. For all the measurements, the Pt electrodes are grounded. But it is also possible to connect a voltage source to them so as to provide solution gating to the Si-NWs. When the $\mathrm{Pt}$ inlet electrode is grounded, the front gate oxide surface is discharged through solution resistance $R_{\text {sol1 }}$, double layer capacitance $C_{\mathrm{dl}}$, and front-gate oxide capacitance $C_{\text {fo }}$. When the Pt outlet electrode is grounded, the gate oxide surface is charged through $R_{\mathrm{sol} 2}, C_{\mathrm{dl}}$, and $C_{\mathrm{fo}}$. When both inlet and outlet electrodes are grounded, charges accumulate on the $\mathrm{Si}-\mathrm{NW}$ gate oxide due to the upstream flow discharge simultaneously by the downstream flow to the Pt electrode through surface conduction.
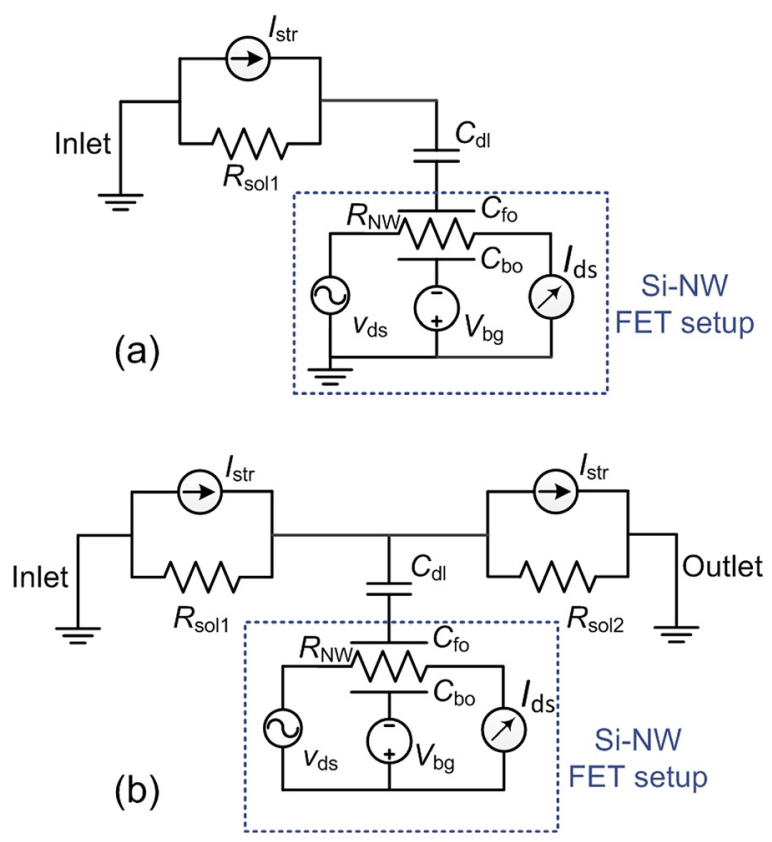

FIG. 2. Equivalent electrical circuits of Si-NW microfluidic system when: (a) inlet only is grounded and (b) both inlet and outlet are grounded.
The electric field inside the microchannel is thus confined by the two Pt electrodes. Therefore, the surface potential of the gate oxide can be maintained constant regardless of varying flow rate.

Figure 3 shows the current measurements from Si-NWs with varying driven pressure. The change of driven pressure can affect the flow rate and the streaming potential over the NW gate oxide, thus the conductance of $\mathrm{Si}-\mathrm{NW}$ sensor changes accordingly. A similar effect can be demonstrated with an external voltage applied to the inlet and outlet electrodes. The driven pressure changes from 100 millibars $\left(V_{\text {flow }} \approx 2.4 \mathrm{~mm} / \mathrm{s}\right)$ to 500 millibars $\left(V_{\text {flow }} \approx 12 \mathrm{~mm} / \mathrm{s}\right)$ can change the surface potential $\psi_{o}$ of Si-NW gate oxide while single side is grounded. The amount of surface potential change $\Delta \psi_{o}$ is dependent on the distance of Si-NW array to the grounding point and the ionic strength of the solution. ${ }^{13}$ The surface potential change $\Delta \psi_{o}$ can be positive or negative with reference electrodes being placed upstream or downstream, respectively. As illustrated in Figs. 3(a) and 3(b), the change of current $\Delta I_{\mathrm{ds}}$ is larger $\left(\Delta I_{\mathrm{ds} 2}=0.7 \mathrm{nA}\right.$ versus $\Delta I_{\mathrm{ds} 1}=0.3 \mathrm{nA}$ ) when inlet is grounded due to the fact that the distance from NW array to the inlet is about three times the distance to the outlet. However, while both sides are grounded, Si-NW sensor is much less reactive to the flow rate change as shown in Fig. 3(c).

Automated sample switching is crucial for realizing multi-sample delivery and sensing. ${ }^{14}$ It can improve sensing efficiency and provide better control of static charge disturbance than manual switching. We use a one-valve system to switch between sample A and sample B. With the outlet Pt tube grounded only, we observe large measured conductance spikes from the NW sensors during the valve switching, both from sample A to sample B, and from sample B to sample A, with about $5 \%$ current change, as illustrated in Fig. 4. The
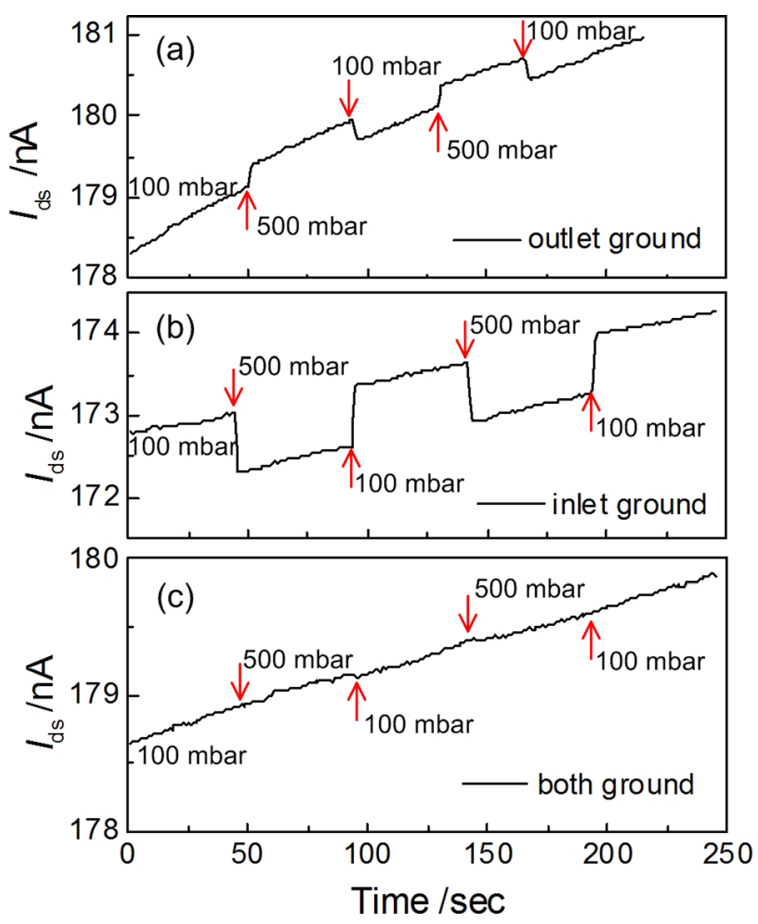

FIG. 3. NW current change due to flow rate variation with (a) outlet grounded, (b) inlet grounded, and (c) both sides grounded. 


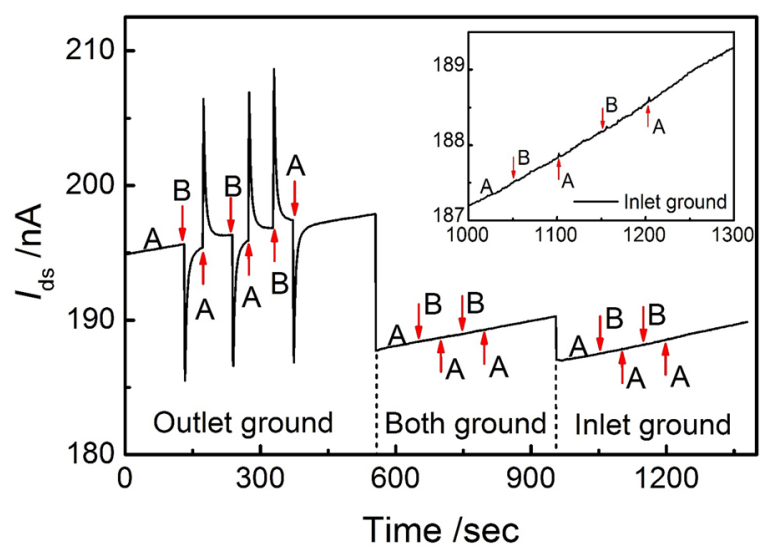

FIG. 4. Valve switching effects with different grounding points.

source of the spikes can be caused by pressure fluctuation during the switching and static charge accumulated on the inner walls of the electrically controlled valve. Releasing of electrostatic charges generated during valve switching to the grounded electrode can affect the downstream charge distribution inside the microchannel. The problem with such current spikes is that it can take a few minutes or more for the current to come back to the baseline through discharging the charges to the ground. The direction of the current spikes can be positive or negative. With the inlet $\mathrm{Pt}$ tube grounded only, we see a great reduction in the switching induced sensor noise. As shown in Fig. 4, it is still possible to resolve the small spike switching events. However, when both inlet and outlet Pt tubes are grounded, the conductance spikes are eliminated at the sample switching times. It should be noted that the variation of current baseline in three stages is due to different grounding points.

In conclusion, we present an effective way to electrically shield the sample solution, which provides stable sample switching for sensors that detect the intrinsic charge of biomolecules. The double $\mathrm{Pt}$ electrodes placed both upstream and downstream can reduce the erroneous sensor signal from either pressure fluctuation or sample switching, and thus can provide stable measurement system for improving detection limit of silicon nanowire biosensors.

This work was supported by NanoNextNL, a micro and nanotechnology consortium of the Government of the Netherlands and 130 partners.

${ }^{1}$ Y. Cui, Q. Q. Wei, H. K. Park, and C. M. Lieber, Science 293(5533), 1289 (2001).

${ }^{2}$ E. T. Carlen and A. van den Berg, Lab Chip 7(1), 19 (2007).

${ }^{3}$ Y. L. Bunimovich, Y. S. Shin, W. S. Yeo, M. Amori, G. Kwong, and J. R. Heath, J. Am. Chem. Soc. 128(50), 16323 (2006).

${ }^{4}$ S. Chen, J. G. Bomer, E. T. Carlen, and A. van den Berg, Nano Lett. 11(6), 2334 (2011).

${ }^{5}$ G. J. Zhang, G. Zhang, J. H. Chua, R. E. Chee, E. H. Wong, A. Agarwal, K. D. Buddharaju, N. Singh, Z. Q. Gao, and N. Balasubramanian, Nano Lett. 8(4), 1066 (2008).

${ }^{6}$ R. Elnathan, M. Kwiat, A. Pevzner, Y. Engel, L. Burstein, A. Khatchtourints, A. Lichtenstein, R. Kantaev, and F. Patolsky, Nano Lett. 12(10), 5245 (2012)

${ }^{7}$ P. R. Nair and M. A. Alam, IEEE Trans. Electron Devices 54(12), 3400 (2007).

${ }^{8}$ E. D. Minot, A. M. Janssens, I. Heller, H. A. Heering, C. Dekker, and S. G. Lemay, Appl. Phys. Lett. 91(9), 093507 (2007).

${ }^{9}$ K. Nishiguchi, N. Clement, T. Yamaguchi, and A. Fujiwara, Appl. Phys. Lett. 94(16), 163106 (2009).

${ }^{10}$ M. Zimmermann, E. Delamarche, M. Wolf, and P. Hunziker, Biomed. Microdevices 7(2), 99 (2005).

${ }^{11}$ T. M. Squires, R. J. Messinger, and S. R. Manalis, Nat. Biotechnol. 26(4), 417 (2008).

${ }^{12}$ B. J. Kirby and E. F. Hasselbrink, Electrophoresis 25(2), 187 (2004).

${ }^{13}$ D. R. Kim, C. H. Lee, and X. L. Zheng, Nano Lett. 9(5), 1984 (2009).

${ }^{14}$ A. De, J. van Nieuwkasteele, E. T. Carlen, and A. van den Berg, Analyst 138(11), 3221 (2013).

${ }^{15}$ S. Chen, J. G. Bomer, W. G. van der Wiel, E. T. Carlen, and A. van den Berg, ACS Nano 3(11), 3485 (2009). 